\title{
ESTUDO COMPARATIVO ENTRE TUBERCULOSOS NÃO INFECTADOS E INFECTADOS PELO HIV, NO DISTRITO FEDERAL
}

Maria do Socorro Evangelista Kusano ${ }^{11}$

\begin{abstract}
RESUMO: Este trabalho se propõe a estudar o grau de associação entre as variáveis: tuberculosos HIV positivos e negativos, em relação à localização e ao tamanho da lesão tuberculosa, e verificar, se há ou não concordância na distribuição dos casos da doença tuberculosa nos grupos masculinos e femininos, infectados ou não pelo HIV, segundo a faixa etária. Analisou-se 78 pacientes de tuberculose/HIV e 2950 casos de tuberculose, entre 1985 a 1992. As informações foram colhidas através dos prontuários e arquivos do Programa de Tuberculose, Hemocentro e Instituto de Saúde do Distrito Federal, Brasília, Brasil. A análise estatística foi realizada através do teste do Qui-Quadrado e do teste Kolmogorov Smirnov. Verificou-se que houve um aumento significativo de tuberculose pulmonar moderada e escavada não cavitária, entre pacientes tuberculosos com HIV, quando comparados com pacientes somente tuberculosos. Os pacientes co-infectados masculinos apresentaram maior incidência de casos, na faixa de idade inferior a 40 anos, quando comparados com os pacientes de tuberculose isolada. As mulheres tuberculosas mostraram um significativo aumento da doença numa faixa etária abaixo de 34 anos, quando comparadas com os homens da mesma idade.
\end{abstract}

UNITERMOS : Epidemiologia - Tuberculose-HIV

\section{INTRODUÇÃO E JUSTIFICATIVA}

A associação de tuberculose em portadores de sorologia positiva para o vírus da Imunodeficiência humana (HIV) tem sido relatado com freqüência em muitos trabalhos realizados nos Estados Unidos, África, América Latina, Caribe, e parte da Ásia nos últimos anos (Rieder et al., 1989); (De Cock "et al"., 1992; CDC, 1992).

Com o aparecimento da pandemia da Síndrome da Imunodeficiência Adquirida (AIDS), novas oportunidades surgiram para a reativação da tuberculose em todas as comunidades inclusive nos paises desenvolvidos.

Nos Estados Unidos, notifica-se cada vez mais casos de tuberculose a partir de 1984 (CHAISSON, R.E.; et al., 1989; RIEDER, H.L.; 1989; COTÊ, T.R. et al., 1990). Um estudo realizado por Cruz (1993), em Santo Domingo, relata que, em crianças com HIV, a tuberculose atingiu 19,4 \%. Segundo a OPAS, a

1 Professora Assistente 1 do Deprtamento de Enfermagem Obstétricia/UNB. 
infecção tuberculosa alcança $62,9 \%$ naquela população, e está associada à transmissão heterossexual pelo HIV, sendo este fator desencadeante do aumento da co-infeç̧ão na República Dominicana (Bol. Of. Sanit. Panam., 1994). Na República Dominicana, em 1991, o percentual acumulado da associação tuberculose-HIV em adultos ficou em torno de $13 \%$ (Bol. Of. Sanit. Panam. 1994).

Na cidade de Buenos Aires, de 1985 a 1991, foram notificados 183 casos da associação tuberculose-HIV. Entre 1986 e 1987, o percentual de soropositivos pelo HIV em pacientes tuberculosos era de 4,8\%: entre 1992 e 1993, esta cifra alcançou $21,0 \%$, permitindo confirmar o alto risco de contrair tuberculose quando infectado pelo HIV (Bol. Of. Sanit. Panam., 1994).

No Brasil, dados do Ministério da Saúde de 1993 (dados preliminares, até a semana 30 , terminada em 31/07/93), mostram que a tuberculose é a terceira ocorrência de doença associada em pacientes notificados com Síndrome da Imunodeficiência Adquirida (SIDA/AIDS). Em 1984, esta associação correspondia a $13,3 \%$ dos casos de AIDS, em julho de 1993, a associação elevou-se para $18,9 \%$. Há uma projeção do Ministério da Saúde de que no período de 1993 a 1995, aparecerão 87 mil casos novos de AIDS, dos quais 20 a $40 \%$ poderão desenvolver tuberculose. Segundo outros autores, a ocorrência de tuberculose em doentes de AIDS está em torno de $22,7 \%$ a $24,2 \%$ dos casos.

A Organização Mundial da Saúde (OMS) estimou que, desde o início da epidemia de AIDS/SIDA até meados de 1993, o número de pessoas infectadas simultaneamente por HIV e Mycrobacterium tuberculosis estaria próximo de 5,1 milhões de pessoas; $95 \%$ destas pessoas, estão em paises em desenvolvimento. A Organização Panamericana de Saúde (OPAS) tinha estimado que, até junho de 1993, o número acumulado de pessoas adultas infectadas pelo HIV, na América Latina e Caribe, seria de 330.000 pessoas (BOL. Sanit. Panam., 1994).

No Distrito Federal, a taxa de incidência de tuberculose aumentou de 23,4 por 100.000 habitantes, em 1987, para 29,0, em 1992. A tuberculose nos portadores de sorologia positiva para o HIV alcançou uma cifra de $9,5 \%$ dos casos em 1992. Quando se compara o número de casos desta co-infecção com os da tuberculose isolada, ano a ano, observa-se a relevância da infecção pelo HIV, levando provavelmente, não só no aumento da incidência da tuberculose no Distrito Federal, como também se supõe, uma alteração nos dados de mortalidade e letalidade (Kusano,1994).

A tuberculose é a única enfermidade ligada ao HIV que é transmissivel à população como um todo, e cujo contágio se dá por via respiratória.

A infecção pelo HIV causa uma progressiva redução na resposta imunicelular do indivíduo, tornando-o predisposto a adquirir uma gama de infecções ditas oportunistas dependentes da imunidade celular. 
A tuberculose é a doença mais freqüente no estágio inicial da infecção causada pelo virus da imunodeficiência humana (HIV). Além disto, a sua gravidade é em geral maior e a apresentação clínica atipica' ${ }^{12}$.

O HIV é um retrovirus humano; para sua sobrevivência e replicação necessita da célula hospedeira e tem tropismo pela células linfocitárias $T$. Os mecanismos imunobiólogicos humanos, responsáveis pelo controle do bacilo de Koch, são mediados pela imunidade celular, que está comprometida pela infecção do HIV.

E importante salientar que células CD4 positivas e macrófagos têm papel central na defesa antibacteriana, e a sua disfunção coloca o paciente HIV soropositivo com maior risco para desenvolver tuberculose primária ${ }^{13}$ ou reativada, em particular nas regiões com alto risco anual de infecção e prevalência da tuberculose. Também é descrito que a infecção ${ }^{14}$ causada pela tuberculose primária, deverá ter um curso mais agressivo em pacientes soropositivo para o HIV. Com a imunodepressão induzida pelo HIV, os casos de tuberculose vêm aumentando e os esforços de controle desta enfermidade têm sido afetados negativamente.

Este estudo tem sua importância para o serviço de atendimento ao paciente co-infectado porque favorece uma maior integração entre clínicos e tisiologistas, com discussão ampla dos casos apresentados por estes pacientes, possibilitando novas opções para aprimoramento das ações de controle da tuberculose. Com a melhoria das condutas clinicas, certamente teremos para os pacientes co-infectados benefícios, como o aumento da esperança de vida, a redução da morbidade e da mortalidade.

Finalmente, estaremos contribuindo para o estudo da doença tuberculosa e infecção pelo HIV no Distrito Federal, ao mesmo tempo que daremos uma visão mais atualizada aos profissionais de saúde das consequências desta associação.

\section{OBJETIVOS}

a) Estudar o grau da associação entre as variáveis: tuberculosos HIV positivos e negativos, em relação à localização e tamanho da lesão tuberculosa;

b) Verificar se há ou não concordância na distribuição de casos da doença tuberculosa nos dois grupos: masculinos e femininos portadores ou não do HIV, segundo faixa etária.

\section{MATERIAL E MÉTODO}

12 Considera-se como padrão radiológico atípico toda a imagem radiológica distinta da imagem típica caracterizada por imagens de hipotransparência de contornos imprecisos, infiltrativos ou nodular de tonalidade baixa com evidência de cavidade localizada nos terços superiores (segmento posterior dos lobos superiores ou segmento superior do lobo inferior).

${ }_{13} \mathrm{O}$ adoecimento por tuberculose imediatamente após a primoinfecção.

${ }^{\text {it }}$ A entrada e desenvolvimento ou multiplicação de um agente infeccioso no organismo de uma pessoa ou animal. 
O presente estudo foi desenvolvido através de pesquisa nos Registros das Unidades de Saúde do Distrito Federal - Coordenações Centrais da Secretária de Saúde, Programa de Controle da Tuberculose, Programa de Controle de DST/AIDS e Hemocentro de Brasília, que desenvolvem ações de controle da tuberculose e AIDS.

O Distrito Federal tem sua área metropolitana estabelecida pelo Decreto $\mathrm{N}^{\circ}$ 10.829, de 14/10/1987, ocupando $5.783 \mathrm{~km}^{2}$. Encontra-se dividido em 12 (doze) Regiões Administrativas (RA), RA I a RA XII, incluindo os núcleos urbanos e rurais.

O setor público do Distrito Federal dispõe de 09 (nove) Hospitais Regionais. 48 (quarenta e oito) Centros de Saúde, 24 (vinte e quatro) Postos de Saúde Rurais e um Hospital de Base ligados à Fundação Hospitalar do Distrito Federal. Há ainda um Hospital Universitário.

\section{População}

A população foi constituida de pacientes portadores de todas as formas de tuberculose, residentes no Distrito Federal há mais de um ano, e portadores ou não do HIV, registrados nas Unidades de Saúde, e que tiveram acesso à assistência médica pública no periodo de 1985 a 1992.

\section{COLETA DE DADOS}

Foi solicitada autorização da Secretária de Saúde (Programa de Controle da Tuberculose e DST/AIDS), da direção dos Hospitais em cada regional, dos chefes dos Centros de Saúde, Hemocentro e do Instituto de Saúde. Estes órgãos detêm a exclusividade da informação sobre a tuberculose, e quase a totalidade dos registros da infecção pelo HIV no Distrito Federal.

Inicialmente, foi realizado um levantamento de todos os casos registrados de tuberculose, em pessoas residentes no Distrito Federal, por meio das seguintes fontes: Coordenação do Programa de Controle da Tuberculose do Distrito Federal; Subsistema de Mortalidade de Tuberculose do Distrito Federal; Laboratório do Instituto de Saúde do Distrito Federal; Hemocentro de Brasília; Centros de Saúde de referência dos pacientes.

Em seguida, foram levantados os registros de pacientes tuberculosos com testes sorológicos positivos para o HIV, referentes a janeiro/85 até dezembro/92.

Os dados desta investigação foram armazenados em banco de dados utilizando-se a linguagem de gerenciamento de banco de dados / DBASE III, no modo interativo, o que possibilitou a ordenação, classificação, cruzamento e emissão de relatórios.

A partir desta triagem foram identificados os pacientes por regional de saúde do Distrito Federal, objetivando verificar se os mesmos preenchiam os critérios pré-estabelecidos no estudo.

- Residentes no Distrito Federal há mais de um ano;

- Diagnóstico de tuberculose estabelecido;

- Portador de tuberculose e do HIV, concomitantemente; 
- Diagnóstico de tuberculose confirmado até a alta.

De todos os registros foram conferidos os dados de identificação, como: nome completo e data de nascimento, a fim de evitar a inclusão de homônimos.

Foram excluídos os seguintes casos:

- os não residentes no Distrito Federal, ou seja, aqueles que não possuíam pelos menos um ano de moradia no Distrito Federal;

- os que iniciaram o tratamento de tuberculose e posteriormente receberam outro diagnóstico;

- os portadores de outras microbacterioses, confirmadas laboratorialmente.

Toda a coleta de dados foi realizada pela autora, no período de 3 de janeiro de 1993 a 30 de junho de 1993.

Os dados relativos à tuberculose foram colhidos através das fichas epidemiológicas encaminhadas, à nível central, pelos servidores das Unidades de Saúde e ambulatórios dos Hospitais, envolvidos no programa de tuberculose. Estes servidores, que prestam serviço ao programa de tuberculose, são treinados sistematicamente para preencherem corretamente os formulários, resultando numa uniformidade de registro de dados.

As informações dos pacientes tuberculosos e com infecção pelo HIV foram levantados através de um instrumento específico, com o objetivo de obter dados relativos ao paciente e outras informações sobre o diagnóstico e tratamento da tuberculose, e diagnóstico da sorologia para o HIV.

Para preenchimento dos instrumentos foram levados em consideração os critérios de classificação de casos, estabelecidos pelos órgãos oficiais e outras recomendações da literatura.

-Critério para classificação de sorologia positiva para o HIV:

Foi considerada sorologia positiva para o HIV aquela que obedecia ao critério estabelecido pelo Ministério da Saúde, que determina a realização de pelo menos dois testes positivos com princípios diferentes. Os testes laboratoriais realizados pelo Hemocentro de Brasília estavam dentro dos critérios e tinham três técnicas distintas: a da Elisa, a imunofluorescência indireta ( IFI) e a imunoimpressão ou Western blot (BRASIL. MINISTÉRIO DA SAÚDE, 1989d; Colaud, 1993).

- Critério para classificação do tipo de exposição ao HIV:

Foi utilizada a classificação segundo o preconizado pelo Serviço de Epidemiologia, Divisão Nacional de Controle de DST/AIDS/MS ( BRASIL. MINISTÉRIO DA SAÚDE, 1989b).

- Categoria de exposição:

Receptor de sangue e hemoderivados; usuário de drogas injetáveis; hemofílico; homossexual; bissexual; heterossexual; transmissão perinatal; transmissão não definida/outra ( caso e/ou categoria não investigada, ou transmissão investigada sem, entretanto, ser caracterizada ou outra categoria definida no investigação, excluindo as acimas relacionadas) e transmissão por fatores múltiplos: homossexual ou bissexual combinado com drogas/ hemofilico, homo-bi/hemofilico, hemofilico/drogas, homo-bi/drogas/transfusão, homo-bi/drogas, homo-bi/transfusão, drogas/transfusão. 


\section{- Critério para classificação de tuberculose: diagnóstico definitivo ou presuntivo:}

Para o diagnóstico da tuberculose, estabeleceu-se a confirmação etiológica por um ou mais dos seguintes métodos: baciloscopia e / ou cultura por meio de aspirado gástrico ou secreção pulmonar; cultura de líquidos orgânicos ou biópsia com exame histopatológico compativel (BRASIL. MINISTÉRIO DA SAÚDE, 1989c).

Nos pacientes sem diagnóstico etiológico estabelecido, utilizou-se os achados epidemiológicos e radiológicos sugestivos da doença, ou os achados clínicos, cuja resposta obtida após a introdução do tratamento específico foi satisfatória, dados esses registrados nos prontuários (BRASIL. MINISTÉRIO DA SAÚDE, 1989; Musella et al., 1990).

As formas pulmonares de tuberculose foram classificadas pelos tisiologistas do Programa da Tuberculose, de acordo com sua extensão, no momento da notificação, segundo critérios da National Tuberculosis Association- USA (BRÓLIO, 1976), com adaptações à necessidades locais.

As formas pulmonares foram classinicadas de acordo com os critérios utilizados pela Coordenação de Programa de Tuberculose do Distrito Federal ${ }^{4}(P C T-D F)$, e incluem: mínima, moderada avançada (cavitária ou não), muito avançada (cavitária ou não) e miliar.

Nas formas extrapulmonares da doença tuberculosa estão: cutânea, ganglionar, ocular, digestiva, pleural, renal, óssea e meningite tuberculosa, entre outras.

\section{DISCUSSÃO}

Para estudar o grau de associação entre as variáveis tuberculosos/HIV positivos e negativos em relação à localização e tamanho da lesão tuberculosa, encontrada em cada grupo de pacientes, foi utilizada o teste do Qui-Quadrado.

Este método estatístico tem por finalidade comprovar se os dois grupos diferem em relação a determinada característica, testando as hipóteses de independência dos grupos em relação à caracteristica analisada (Ho), contra a hipótese de não independência $(\mathrm{Ha})$.

A estatística do teste é dada por:

\footnotetext{
${ }^{+}$No Distrito Federal, a tuberculose miliar, apesar de ser uma forma disseminada, está incluída entre as pulmonares porque o diagnóstico é feito em geral pela lesão deste orgão. Inversamente, observa-se que a tuberculose pleural está incluida nas formas extrapulmonares, por se comportar epidemiologicamente como uma forma de tuberculose pós-primária e não infectante.
} 


$$
x^{2}=\frac{\sum\left(f_{o}-f_{e}\right)^{2}}{f_{e}}
$$

$f_{0}=$ Valor observado

$f_{e}=$ Valor esperado

Sob Ho, mostra-se que $X^{2}$ tem distribuição Qui-Quadrado com g.I = (I-1). (c1), onde I é o número de linhas (características) e $c$ o número de colunas (grupos).

Aplicando-se a estatistica neste estudo, obteve-se como resultado um valor de $X^{2}=29,59$. Com o nivel de significância estabelecido de $5 \%$ e 3 graus de liberdade, o valor de $X^{2}$ tabelado é de 7,82.

Como o valor de $\mathrm{X}^{2}$ calculado é maior do que o valor tabelado (distribuição de Qui-Quadrado com g.l.), rejeitamos Ho ao nivel de significância de 5\%, como pode ser observado na figura 1 .

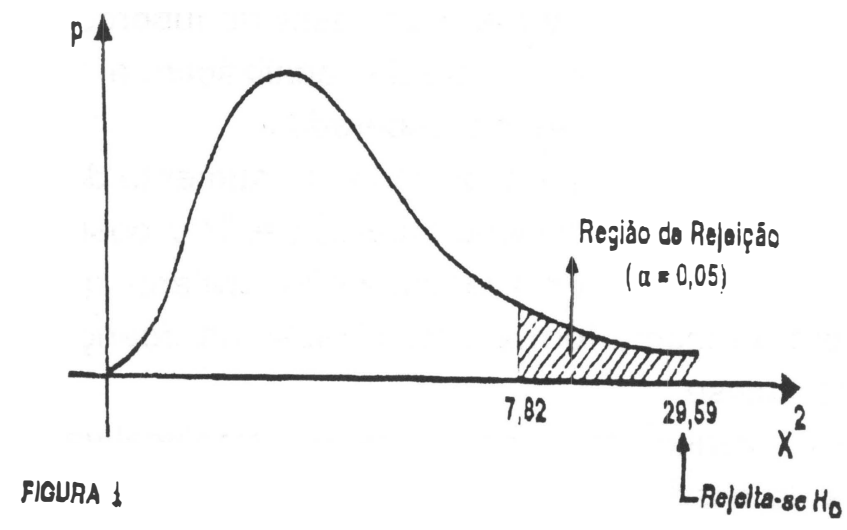

Esta prova mostra então que existe uma diferença, em relação à localização e tamanho da lesão tuberculosa entre os grupos HIV positivos e negativos.

TABELA 1: Freqüência de casos entre pacientes tuberculosos com sorologia positiva e negativa para o HIV, segundo 
localização e tamanho da lesão, no Distrito Federal, de 1985 a 1992.

\begin{tabular}{l|r|r|r}
\hline \multirow{2}{*}{ Localização e Tamanho da Lesão } & \multicolumn{2}{|l|}{ TUBERCULOSOS } & \multirow{2}{*}{ TOTAL } \\
\cline { 2 - 4 } & \multicolumn{1}{|l|}{ HIV + } & HIV - & 684 \\
\hline $\begin{array}{l}\text { Pulmonar } \\
\quad \text { Mínima }\end{array}$ & $14(17,6)$ & $670(0666,4)$ & 1281 \\
$\begin{array}{l}\text { Moderada e Avançada } \\
\quad \text { Cavitária }\end{array}$ & $19(33,0)$ & $1262(1248,0)$ & 373 \\
$\quad$ Não-Cavitária & $24(09,6)$ & $349(0363,4)$ & 690 \\
\hline Extrapulmonar & $21(17,8)$ & $669(0672,2)$ & 3028 \\
\hline TOTAL & 78 & 2950 & \\
\hline
\end{tabular}

TABELA 2 - Desvios observados e esperados entre pacientes tuberculosos com sorologia positiva e negativa para HIV, segundo localização e tamanho da lesão, no Distrito Federal, de 1985 a 1992.

\begin{tabular}{l|c|c}
\hline \multirow{2}{*}{ Localização e Tamanho da Lesão } & \multicolumn{2}{|c}{ TUBERCULOSOS } \\
\cline { 2 - 3 } Pulmonar Hínima & HIV + & HIV - \\
Moderada e Avançada & $-4(0,88)$ & $4(0,02)$ \\
Cavitária & $-14(5,93)$ & $14(0,15)$ \\
Não-Cavitária & $16(28,44)$ & $-16(0,70)$ \\
\hline Extrapulmonar & $2(0,22)$ & $-2(0,005)$ \\
\hline
\end{tabular}

As Tabelas 1 e 2 mostram o número de casos de tuberculose com sorologia positiva e negativa para o HIV, segundo localização e tamanho da lesão tuberculosa e os desvios observados e esperados.

Foi possivel observar que está ocorrendo um aumento do número de casos que supera o esperado, na associação tuberculose/HIV positivo, com relação a tuberculose pulmonar moderada e avançada não cavitária, quando comparados com pacientes com a mesma forma e localização da doença e com pacientes somente com tuberculose.

Por outro lado, verifica-se uma diferença significativamente menor no número de casos da associação tuberculose/HIV positiva, quando relacionada à tuberculose pulmonar moderada e avançada cavitária, em comparação com grupo de pacientes tuberculosos pulmonares sem infecção pelo HIV.

Os trabalhos publicados na literatura nacional e internacional referem uma proporção de casos de tuberculose pulmonar, sem a presença de cavidades entre os pacientes tuberculosos e positivos para infecção pelo HIV, tendo concordância com estudo realizado no Distrito Federal (Chaisson \& Slutkin, 
1989; Joint Tuberculosis COMMITTEE OF THE BRITISH THORACIC SOCIETY, 1992; Côte et al., 1990; Barnes, et al., 1991; Gonçalves et al., 1987).

Um estudo realizado na Flórida (Rieder et al., 1989) mostra um grande número de tuberculose não cavitária, entre pacientes com doença SIDA/AIDS.

A OPAS refere em trabalhos de Pitchenik, (1984) e Suderan (1986) que os resultados de exames radiológicos de tórax nos pacientes co-infectados são atípicos e com baixa apresentação de cavitações (12.6 \%). Shafer (1991) no seu estudo, encontrou apenas $17 \%$ dos pacientes sem cavitações. Cuesta (1989) refere também uma maior incidência de tuberculosos com cavitações entre pacientes com HIV negativos; encontrou também dados estatísticos significantes em pacientes HIV positivos, sem cavitações.

A prova de Kolmogorov-Smirnov foi também utilizada este teste, comprova se nas duas amostras os casos estão tendo a mesma distribuição $(\mathrm{Ho})$, ou seja, se a distribuição dos casos nos dois grupos são concordantes ou não. $O$ método consiste em aplicar para cada amostra uma distribuição de freqüência cumulativa nos mesmos intervalos, é subtraida uma função da outra e a prova focaliza o maior dos desvios observados (DMAX).

A seguir, aplica-se a estatistica correspondente $\alpha$ de $0,05 \%$ :

$$
\text { DMS }=1,36 \cdot \sqrt{\frac{n_{1}+n_{2}}{n_{1} \cdot n_{2}}}
$$

Encontrando-se o valor de DMS, este é comparado com o observado (DMX) a fim de tomarmos a decisão, rejeitar ou não Ho. Se DMS $\geq$ DMAX, rejeitamos Ho.

Quando aplicamos o teste no grupo masculino ( com tuberculose e HIV positivo ou negativo), segundo a faixa etária, obteve-se como resultado:

DMS $<$ DMAX, ou $0,165<0,19$ por isso, não rejeitamos Ho.

$\mathrm{Na}$ Tabela 3, quando se comparou o grupo masculino (tuberculose com ou sem sorologia positiva para o HIV) e a faixa etária, observou-se que houve um significativo aumento do número de casos, que supera o esperado, em idades menores de 40 anos, no grupo de tuberculose com HIV. 
significativo aumento do número de casos, que supera o esperado, em idades menores de 40 anos, no grupo de tuberculose com HIV.

TABELA 3 - Incidência de portadores de tuberculose do sexo masculino com sorologia positiva e negativa para HIV, segundo a faixa etária, no Distrito Federal, de 1985 a 1992

\begin{tabular}{|c|c|c|}
\hline \multirow[b]{2}{*}{ FAIXA ETARIA } & \multicolumn{2}{|c|}{ MASCULINO } \\
\hline & TUBERCULOSEIHIV & TUBERCULOSE \\
\hline $00-04$ & $x_{-1}$ & $92(0,05)$ \\
\hline $05-09$ & - & $64(0,09)$ \\
\hline $10-14$ & - & $30(0,11)$ \\
\hline $15-19$ & $01(0,01)$ & $102(0,17)$ \\
\hline $20-24$ & $07(0,11)$ & $172(0,27)$ \\
\hline $25-29$ & $12(0,28)$ & $210(0,39)$ \\
\hline $30 \div 34$ & $16(0,51)$ & $193(0,51)$ \\
\hline 35,39 & $19(0,77)$ & $226(0,64)$ \\
\hline $40-44$ & $11(0,92)$ & $154(0,73)$ \\
\hline $45-49$ & $03(0,97)$ & $104(0,79)$ \\
\hline $50 e^{+}$ & $02(1,00)$ & $339(1,00)$ \\
\hline TOTAL & 70 & 1686 \\
\hline
\end{tabular}

$\mathrm{Na}$ aplicação do teste no grupo com tuberculose isolada, ou seja, sem infecção pelo HIV (Tabela 4), distribuido por faixa etária e sexo, observou-se que DMS $<$ DMAX ou $0,0502<0,14$; então aceitamos Ho.

É possivel descrever que está ocorrendo um significativo aumento no número de casos, nos pacientes do sexo feminino com tuberculose, nas faixas etárias mais jovens, até 34 anos, quando comparados com os do sexo masculino com tuberculose isolada e mesma faixa etária.

TABELA 4 - Distribuição dos casos de tuberculose, segundo faixa a etária e o sexo, no Distrito Federal, de 1985 a 1992.

\begin{tabular}{|c|c|c|}
\hline \multirow[b]{2}{*}{ FAIXA ETARIA } & \multicolumn{2}{|c|}{ TUBERCULOSE } \\
\hline & MASCULINO & FEMININO \\
\hline 0.4 & 92 & 155 \\
\hline 5.9 & 64 & 56 \\
\hline $10-14$ & 30 & 51 \\
\hline $15-19$ & 102 & 79 \\
\hline 20.24 & 172 & 173 \\
\hline 25.29 & 210 & 167 \\
\hline 30.34 & 193 & 181 \\
\hline 35.39 & 226 & 85 \\
\hline $40 \quad 44$ & 154 & 73 \\
\hline 45.49 & 104 & 80 \\
\hline $50 e+$ & 339 & 210 \\
\hline TOTAL & 1686 & 1304 \\
\hline
\end{tabular}


TABELA 5 - Distribuição dos casos de tuberculose associados à infecção pelo HIV, segundo a faixa etária e o sexo, no Distrito Federal, de 1985 a 1992.

\begin{tabular}{|c|c|c|}
\hline \multirow[b]{2}{*}{ IAIXA ISTARIA } & \multicolumn{2}{|c|}{ TUBSRCUI OSIIHIV } \\
\hline & MASCLILINO & ILMANINO \\
\hline $0(1) 4$ & - & 2 \\
\hline $05-109$ & - & - \\
\hline $10-14$ & 7 & - \\
\hline 15.19 & 1 & 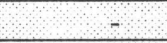 \\
\hline $20-24$ & 7 & $\rightarrow$ \\
\hline $25-29$ & 12 & 1 \\
\hline $30-34$ & 16 & 3 \\
\hline $35-39$ & 18 & - \\
\hline $40-44$ & 11 & 1 \\
\hline $45-49$ & 3 & 1 \\
\hline $5 t) e+$ & 2 & - \\
\hline IOIAI & 70 & 8 \\
\hline
\end{tabular}

Aplicando-se o teste no grupo de pacientes com associação tuberculose e HIV positivo, segundo a faixa etária e a sexo, encontrou-se DMS>DMAX, ou seja, 0,236<0,507 sendo então rejeitado Ho (Tabela 5).

Conclui-se que, entre as pessoas com tuberculose e positivas para o HIV, não houve diferença significativa, quando comparadas por sexo e faixa etária.

TABELA 6 - Incidência de portadores de tuberculose no sexo feminino, com sorologia positiva e negativa para HIV, segundo a faixa etária, no Distrito Federal, de 1985 a 1992.

\begin{tabular}{|c|c|c|}
\hline \multirow[b]{2}{*}{ IAIXA WTARIA } & \multicolumn{2}{|c|}{ TIMININO } \\
\hline & TUBBERC ULOSSTHUV & IUA3IIRCOLOSA \\
\hline$(0)-04$ & 2 & 155 \\
\hline $05-199$ & 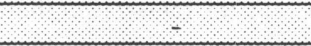 & 56 \\
\hline $10-14$ & - & 51 \\
\hline $15-19$ & - & 79 \\
\hline $20-24$ & 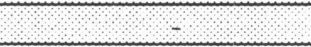 & 173 \\
\hline $25-29$ & 1 & 161 \\
\hline 3()$, 34$ & 3 & 181 \\
\hline $35-39$ & - & 85 \\
\hline 40) 44 & 1 & 73 \\
\hline $45-49$ & 1 & 80 \\
\hline $50 \mathrm{et}$ & - & 210 \\
\hline TOIAL, & 8 & 1304 \\
\hline
\end{tabular}

O teste de Kolmogorov-Smirnov também foi aplicado no grupo feminino com associação de tuberculose e HIV positivo e negativo (Tabela 6), segundo 
a faixa etária. Encontrou-se a DMS >DMAX, ou, 0,105-0.482. sendo rejeitado Ho.

$\mathrm{Na}$ comparação realizada neste grupo feminino com tuberculose e sorologia positiva e negativa para o HIV, não se obteve resultado significativo. quando distribuido por faixa etária e sexo.

\title{
CONCLUSÕES
}

Pode-se observar com este estudo que houve um aumento significativo de tuberculose pulmonar moderada e avançada não cavitária, entre pacientes tuberculosos com HIV, quando comparamos com o grupo de pacientes somente com tuberculose.

No grupo de tuberculosos com HIV e sexo masculino, estes apresentaram maior freqüência de casos, na faixa de idade inferior a 40 anos, quando comparadas com o grupo de pacientes com tuberculose isolada

Nos pacientes só com tuberculose, houve um significativo aumento de casos entre as mulheres, com idade abaixo de 34 anos, quando comparadas com os homens nas mesmas faixas etárias.

Entre as pessoas co-infectadas, não houve diferença significativa, quando comparados por sexo e faixa etária.

A comparação realizada no grupo feminino de tuberculose, com sorologia positiva e negativa para o HIV, não se obteve resultado significativo, quando distribuido por faixa etária e sexo.

\begin{abstract}
The main aim of this paper is to study the degree of association between the variables: HIV positive and negative tuberculosis patients, in relation to the location and extension of tuberculosis lesion and also to observe whether there is or not an agreement in distribution of tuberculosis disease between male and female groups, infected or not by HIV, according to their age. Seventy-eight tuberculosis/HIV positive and 2950 tuberculosis patients without HIV between 1985 and 1992 have been analysed. The data have been collected directly from the files kept at Hemocentro and Instituto de Saude do Distrito Federal. Brasilia-DF, Brazil. Statistical analysis using the X-squared and Kolmogorov Smirnov's tests have been applied. It has been observed a significant increase in the cases of pulmonary cavity and not cavity moderate tuberculosis among the fuberculosis/HIV positive patients when compared with the ones only infected with tuberculosis. In the age group below 40 years old. the male presented a higher incidence of cases when compared with the ones without HIV. In the age group below 34 years old, women showed a significative increase of the disease. when compared to men in the same age group.
\end{abstract}

KEYWORDS: Epidemiology - Tuberculosis - HIV. 


\section{BIBLIOGRAFIA}

1. BARNES, P.F.; BLOCH, A.B.; DAVIDSON, P.T. et al. Tuberculosis in patients with human immunodeficiency virus infection. N.Engl.J. Med.; v. 324. n.23. p.1644-50, 1991.

2. BRASILIA. MINISTÉRIO DA SAÚDE. Manual de Aconselhamento/AIDS. Brasilia, 1989a.

3. MINISTÉRIO DA SAÚDE. Boletim Epidemiológico da AIDS. Brasília, ano III, n.2. Semana epidemiológica 31-35, 1989b.

4. MINISTÉRIO DA SAÚDE. Controle da Tuberculose: Uma proposta de Integração Ensino-Serviço. 2 ed. rev. Rio de Janeiro, 1989c.

5 MINISTÉRIO DA SAÚDE. Manual de Normas para o Controle da Tuberculose. Brasilia, 1989d.

6. BRÓLIO, R.; FILHO, M.T.L. Tuberculose pulmonar. In: VERONESI, R. Doenças Infecciosas e parasitárias. $6^{\text {a }}$ ed. Rio de Janeiro: Guanabara Koogan, 1976, p.317-38.

7. CDC. National action plan to combat multidrug-resistant tuberculosis. MMWR, v.41, n.RR-11, p. 5-8, 1992.

8. CHAISSON, R.E.; SLUTKIN, G. Tuberculosis and the human immunodeficiency virus infection. J. Infect. Dis., v.159, n.1, p.96-100, 1989.

9. COLAUD, J. P. In: Doenças Sexualmente Transmissiveis e AIDS. Porto Alegre. Artes Médicas, p.277-86, 1993.

10. COTÉ, T. R.; NELSON, M. R.; ANDERSON, S. P. et al. The present and the future of AIDS and tuberculosis in Illinois. AJPH. v.80, n.8, p.950-3, 1990.

11. CUeStA, F.L.; HERRERO, C.P.C.; AGUAdO, C.G. "at al." Tuberculosis y infección por HIV: estudio de 50 casos. Rev. Clin. Esp., v. 184, n.5, p.230-33, mar. 1989. 
12. De COCK. K. M.; SORO, B.; COULIBALY, I. M. et al. Tuberculosis and HIV infection in sub-saharan Africa. JAMA, v.268, n.12; p.1581-6, 1992.

13. ESPINAL, M. SIDA. Infección por HIV y tuberculosis: su asociación e impacto en la República Dominicana. Bol. Of. Sanit. Panam., v.116. n. 5,1994 .

14. GARCIA, M. L.; GOMEZ, J. L.; SANCHO, C.G. et al. Epidemiologia del SIDA y la tuberculosis. Bol. Of. Sanit. Panam. v.116, n.6, 1994.

15. GONÇALVES, A.J.R.; MENEZES, J.A.; CUNHA, R. Q. et al. Tuberculose e Sindrome de Imunodeficiência Adquirida. Arq. Bras. Med., v.61, n.6, p.385-8, 1987.

16. JOINT TUBERCULOSIS COMMITTEE OF THE BRITISH THORACIC SOCIETY. Guidelines on the managemente of tuberculosis and HIV infection in the United Kingdom. BMJ, v.304, p.1231-3, 1992.

17. KANTOR, I. N.; ASTARLOA, L.; MONTANER, L.J.G. Asociación entre tuberculosis e infección por virus de la inmunodeficiencia humana en la Argentina. Bol. Of. Sanit.Panam. v.11, n.4, 1994.

18. KUSANO, M.S.E. Características clínicas e epidemiológicas dos portadores de tuberculose e sorologia positiva para 0 virus da imunodefiência humana (HIV) no Distrito Federal. Dissertação (Mestrado).

19. MUSELLA, R. M.; CASTAGNINO, J.P.; WEBER, L. et al. Asociación de la infección por el virus de la imunodeficiencia humana (HIV) y tuberculosis. Medicina. v. 50, n.1, p.3-8. 1990.

20. RIEDER, H.L.; CAUTHEN, G.M.; BLOCH, A.B. et al. Tuberculosis and Acquired Immunodeficiency Syndrome- Florida. Arch. Inter. Med. v.149, p.128-73, 1989.

21. ZACARIAS, F.; GONZALEZ, S. R.; CUCHÖ, P.et al. El SIDA y su interacción con la tuberculosis em América Latina y Caribe. Bol. Of. Sanit. Panam. v. 116, n. 3, 1994. 